\title{
ANÁLISE DO CONTORNO DA FACE PROXIMAL OBTIDO EM RESTAURAÇÕES CLASSE II DE RESINA COMPOSTA UTILIZANDO-SE DOIS TIPOS DIFERENTES DE MATRIZES METÁLICAS
}

\author{
PROXIMAL CONTOUR ANALYSIS OBTAINED FROM CLASS II IN COMPOUND RESIN RESTORATIONS USING TWO \\ DIFFERENT TYPES OF METALLIC MATRICES.
}

\author{
Tatiana Andrade Costa* \\ Ricardo Raitz ** \\ Luis Carlos Belan *** \\ Maurício Rufaiel Matson **
}

\begin{abstract}
RESUMO
Introdução: $\mathrm{O}$ contorno anatômico proximal em restaurações depende muito do tipo de matriz utilizada. $\mathrm{O}$ objetivo desse estudo "in vitro" é analisar o contorno proximal em cavidades do tipo classe II restauradas com resina composta, utilizando-se dois tipos de matrizes metálicas, a convencional (tipo Tofflemire) e a seccionada (Unimatrix). Métodos: Foram selecionados 20 dentes com cavidades mésio-oclusal padronizadas com completa perda do contato proximal. Os dentes foram divididos em dois grupos e restaurados utilizando-se no Grupo 1 a matriz Tofflemire e no Grupo 2, a matriz seccionada Unimatrix. Posteriormente, os dentes foram fotografados e o contorno proximal foi comparado, um a um, com o dente-padrão (hígido). Foram marcados três pontos no dente-padrão que serviram de referência para a medição do contorno. Essa distância foi medida entre os pontos em milímetros para avaliar se o sistema de matriz proporcionou sobrecontorno ou subcontorno. As diferenças foram anotadas em uma planilha eletrônica e os valores submetidos à ANOVA e as diferenças entre os pontos foram analisadas com o teste NewmanKeuls. Resultados: As diferenças entre os pontos foram estatisticamente significantes nos dentes do grupo 1, mas não nos dentes do Grupo 2, mostrando-se que as restauraçôes dos dentes do Grupo 1 não tiveram um contorno adequado, ao contrário do Grupo 2. Conclusôes: As diferenças entre as médias não são ocasionais, mas relacionadas aos sistemas de matriz. O sistema de matriz seccionada proporciona um contorno anatômico mais adequado quando comparado ao sistema de matriz convencional.
\end{abstract}

DESCRITORES: Restauraçáo dentária permanente • Resinas compostas

\section{ABSTRACT}

Introduction: The anatomical proximal contour in dental restorations depends on the type of matrix that is used. The aim of this "in vitro" study is to analyze the proximal contour of Class II in composite resin restored cavities, using two types of metal matrix, the conventional (Tofflemire) and the sectioned one (Unimatrix). Methods: 20 teeth were selected, all of them containing a mesio-occlusal cavity with complete loss of the proximal contact. The teeth were divided into two groups and restored using the Tofflemire matrix in the Group 1 and the sectioned matrix (Unimatrix) in the Group 2. Subsequently, the teeth were photographed and the proximal contour was compared, one by one, with the pattern tooth (healthy). Three points were marked on the pattern tooth and were used as a reference for measuring the contour. This distance was measured in millimeters between the points to evaluate whether the matrix caused excessive contour or insufficient one. Differences were recorded in the Excel program and the values were submitted to ANOVA and differences between points were analyzed with the Newman-Keuls test. Results: Differences between points were statistically significant in the teeth of Group 1, but not in the teeth of Group 2 , showing that the teeth restorations in Group 1 did not present an appropriate contour, and that was not true for Group 2. Conclusions: The differences between the means are not occasional, but are related to the matrix systems. The sectional matrix provides better contour when compared to the conventional matrix.

DESCRIPTORS: Dental restorations, permanent $\bullet$ Composite resins

\footnotetext{
* Especialista em Dentística Restauradora pela ABENO-SP. maumatson@gmail.com

** Professores titulares do curso de mestrado em Odontologia/Bioodontologia da Universidade Ibirapuera.

*** Professor do curso de Especialização em Dentística Restauradora da FUNDECTO.
} 
Costa TA, Raitz R, Belan LC, Matson MR. Análise do contorno da face proximal obtido em restaurações classe ii de resina composta utilizando-se dois tipos diferentes de matrizes metálicas. Revista de Odontologia da Universidade Cidade de São Paulo 2009 jan-abr; 21(1): $31-7$

\section{INTRODUÇÃO}

O contato liso e firme entre os dentes adjacentes tem um importante papel fisiológico em manter a saúde dos tecidos orais. Contatos firmes evitam a impactaçáo de alimentos (Keogh e Bertolotti ${ }^{3}$, 2001). Pontos de contato deficientes podem resultar em impactação alimentar, que levaria a um acúmulo de placa em longo prazo. Ao contrário das restauraçóes de amálgama, nas restauraçóes de resina composta em dentes posteriores, o contato proximal e o contorno anatômico da face proximal náo podem ser obtidos exclusivamente por meio da pressão de condensação (Chain e Baratieri ${ }^{1}$, 1998; Klein et al. ${ }^{4}$, 2002), a despeito da existência de resinas condensáveis.

Diferentes tipos de técnicas vêm sendo desenvolvidas para melhorar os contatos proximais, como: préencunhamento, utilização de matrizes ultrafinas e alguns dispositivos especiais que ajudam na polimerização e na pressão do material restaurador contra a matriz (Lighttip, Contact-Pro, Incertos cerâmicos) (Keogh e Bertolotti $\left.{ }^{3}, 2001\right)$.

O sistema de matriz convencional (Tofflemire) consiste em uma tira metálica fina e lisa colocada circunferencialmente ao dente e presa em um porta-matriz. Com esse sistema, fica difícil recriar o contorno anatômico proximal do dente com resinas compostas, pois esse contorno apresenta uma convexidade oclusalmente e uma concavidade gengivalmente (Lowe 5 , 2004). O sistema de matriz seccionada apresenta uma concavidade na face interna para dar forma à face proximal da restauração. Essa matriz é retida por um anel retentor que além de manter a matriz em posição, causa uma separação entre o dente afetado e o dente adjacente (Lowe ${ }^{5}$, 2004).

O presente trabalho tem como objetivo comparar in vitro 2 sistemas de matriz metálica (matriz convencional e matriz seccionada) no que se refere ao contorno da face proximal em restauraçóes classe II com resina composta.

\section{MÉTODOS}

Foram selecionados 20 dentes de um manequim Odontológico (PRODENS ${ }^{\circledR}$ ) primeiros molares inferiores esquerdos, com cavidades mésio-oclusal (MO) de largura vestíbulo-lingual de $3,5 \mathrm{~mm}$, abrangendo a metade da face oclusal dos dentes no sentido mésio-distal. Essas cavidades foram padronizadas com completa perda do contato proximal. Previamente à restauração, os dentes foram colocados em um manequim (PRODENS ${ }^{\oplus}$ ), com todos os outros dentes íntegros.

Os dentes foram divididos em dois grupos com $\mathrm{n}=10$. Ambos os grupos de dentes foram restaurados com a resina composta $\mathrm{P} 60^{\circ}$, do fabricante $3 \mathrm{M}^{\oplus}$. Para ambos os grupos foram usadas cunhas de madeira com formato triangular. A técnica restauradora para inserção da resina em ambos os casos foi a incremental, com incrementos de resina de no máximo $2 \mathrm{~mm}$ e polimerizaçáo intermediária de 20 segundos para cada incremento.

No Grupo 1, a matriz utilizada foi a metálica tipo Toflemire, no. 01 , de espessura $0,050 \mathrm{~mm}$, do fabricante Dental Suply Company ${ }^{\oplus}$, retida por um porta matriz Toflemire do fabricante Jon ${ }^{\oplus}$, com cunha de madeira interproximal.

No Grupo 2, foi utilizada a matriz metálica seccionada da marca Unimatrix ${ }^{\oplus}$ do fabricante TDV $^{\oplus}$. Esse sistema baseia-se em uma matriz seccionada com uma concavidade interna para dar forma à parede proximal das restauraçóes. Seu princípio de funcionamento é baseado numa força oposta exercida no contato interproximal pelo anel retentor em contato com os dentes.

Depois dos dentes serem restaurados, estes foram colocados um a um em um soquete de silicona previamente preparado, e fotografados sem que este soquete

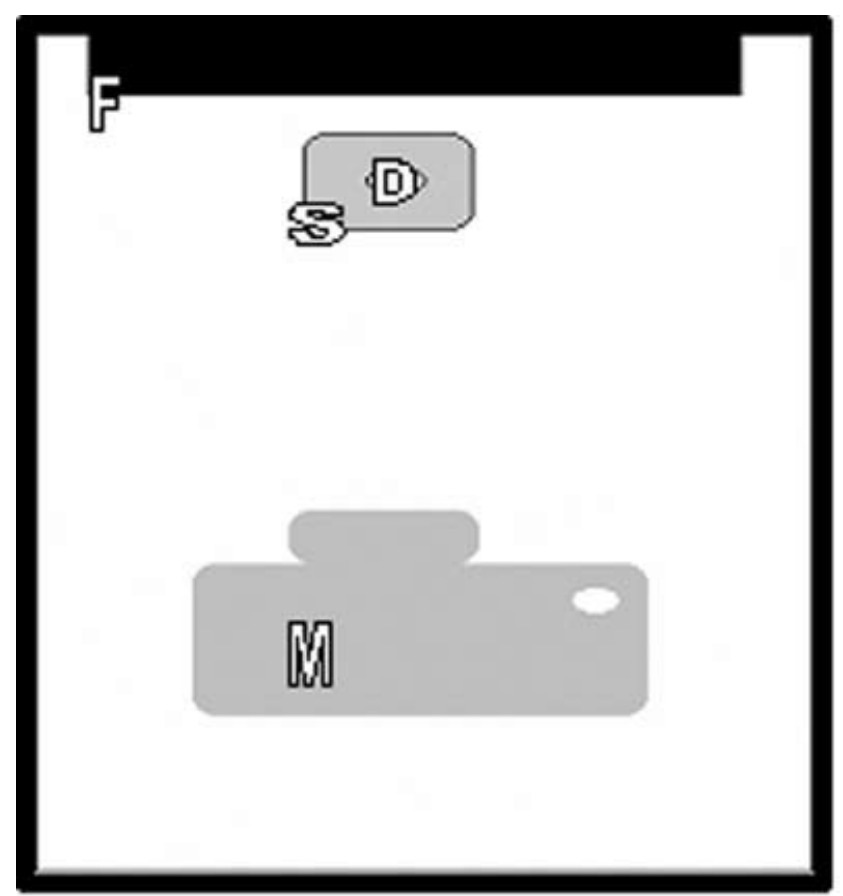

Figura 1: Esquema para fazer as fotografias dos dentes. Onde M: máquina fotográfica, S: soquete, D: dente e F: fundo preto. 
Costa TA, Raitz R, Belan LC, Matson MR. Análise do contorno da face proximal obtido em restaurações classe ii de resina composta utilizando-se dois tipos diferentes de matrizes metálicas. Revista de Odontologia da Universidade Cidade de São Paulo 2009 jan-abr; 21(1): $31-7$

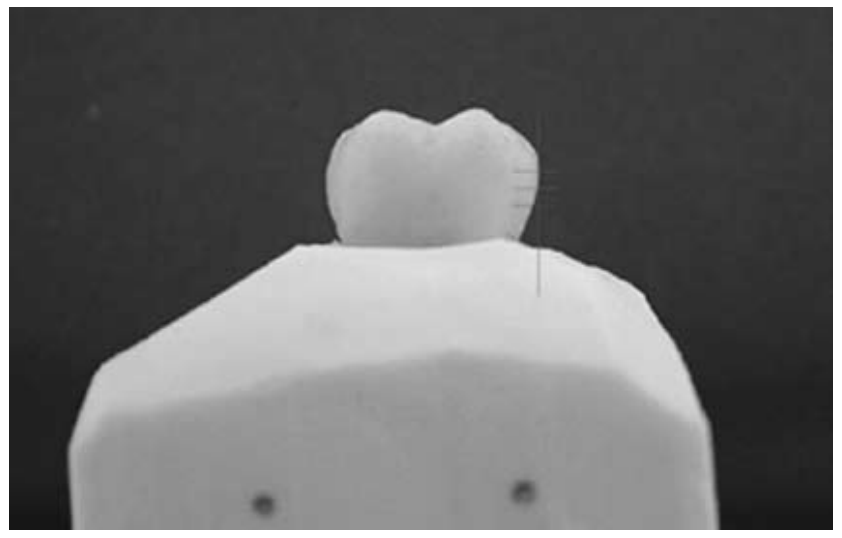

Figura 2A: Gabarito com os pontos marcados na face mesial.

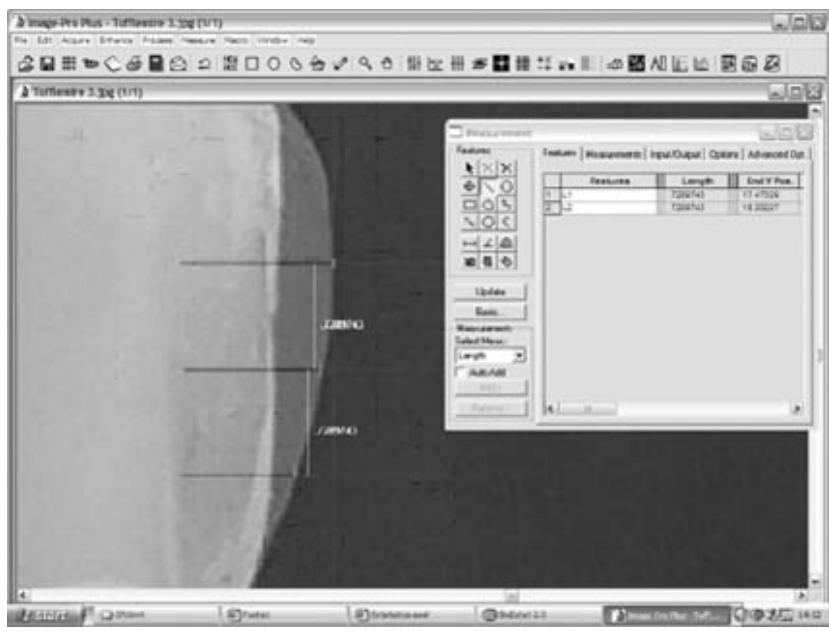

Figura 2B: Distância entre os pontos

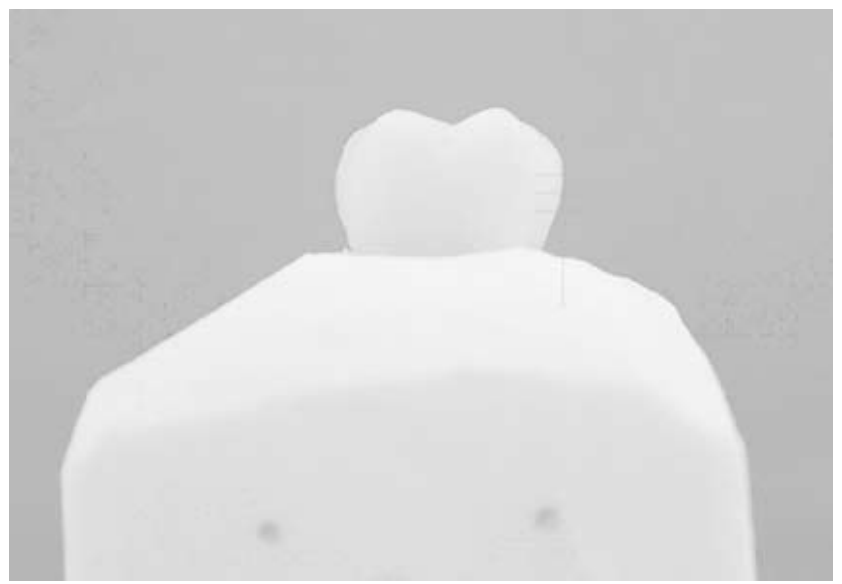

Figura 3: Foto do gabarito tratado no Adobe ${ }^{\circledR}$ Photoshop Acrobat

ou a máquina fotográfica tivesse alguma movimentação. Para que essa movimentação não ocorresse, o soquete e a máquina foram estabilizados com fita adesiva sobre uma base rígida e as fotos foram tiradas com a mesma apro-

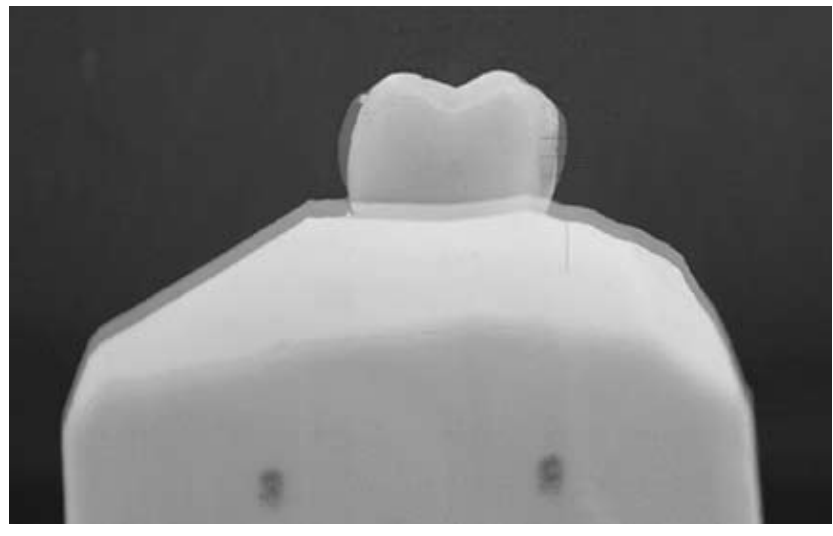

Figura 4: Foto do gabarito sobreposta ao dente do grupo 1 (Tofflemire)

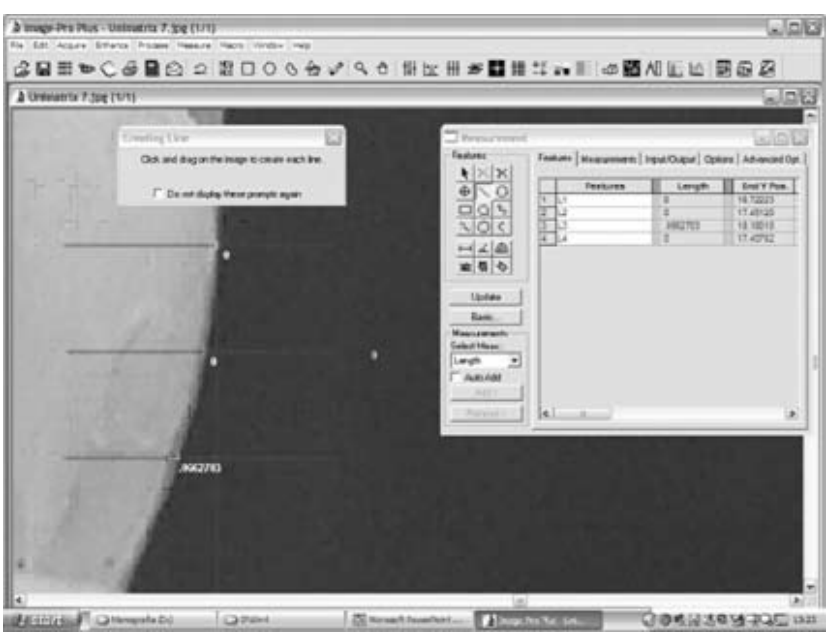

Figura 5: Distância entre os pontos do grupo 2 (Unimatrix)

ximação e em sequência, conforme a Figura 1. O dentepadráo hígido (controle) também foi posicionado no soquete e fotografado para as comparaçôes. Foram feitas duas marcas na base do soquete com distância de $10 \mathrm{~mm}$ para posterior calibração do software para as medições.

Tomou-se como dente-padrão o primeiro molar inferior esquerdo, "hígido", sem preparo, do mesmo fabricante dos demais (PRODENS ${ }^{\circ}$ ). Os dentes foram restaurados por um mesmo operador e as mensuraçóes feitas por meio de fotos e comparadas com a foto do dente-padrão (usado como referência ou gabarito). $\mathrm{O}$ gabarito foi feito marcando-se três pontos na face mesial do dente (Figura 2A) sendo que o ponto 1 foi feito no equador anatômico da regiáo proximal; o ponto 2 a $0,73 \mathrm{~mm}$ do ponto 1 no sentido cervical; e o ponto 3 a $0,73 \mathrm{~mm}$ do ponto 2 no sentido cervical (Figura 2B). Esses pontos foram feitos utilizando-se o software de imagens Adobe ${ }^{\odot}$ Photoshop Acrobat 7.0.1. Os pontos 1, 2 e 
Costa TA, Raitz R, Belan LC, Matson MR. Análise do contorno da face proximal obtido em restaurações classe ii de resina composta utilizando-se dois tipos diferentes de matrizes metálicas. Revista de Odontologia da Universidade Cidade de São Paulo 2009 jan-abr; 21(1): $31-7$

Tabela 1 -Diferença entre os pontos na planilha eletrônica (Excel $($ R)

\begin{tabular}{cccccc}
\hline \hline T1 & T2 & T3 & U1 & U2 & U3 \\
\hline 0.3977 & 0.3534 & 0.2651 & 0 & 0.0663 & 0.0663 \\
0.3534 & 0.3313 & 0.1988 & 0.3534 & 0.3976 & 0.3976 \\
0.3534 & 0.3313 & 0.1767 & 0.243 & 0.3093 & 0.3093 \\
0.5302 & 0.5081 & 0.3755 & 0.0884 & 0.1325 & 0.1546 \\
0.5302 & 0.4197 & 0.4639 & 0.1325 & 0.1325 & 0.1325 \\
0.4418 & 0.4639 & 0.3534 & 0.0884 & 0.1104 & 0.0884 \\
0.5081 & 0.4639 & 0.3093 & 0 & 0 & 0.0662 \\
0.4639 & 0.486 & 0.3534 & 0.2209 & 0.2872 & 0.2651 \\
0.4639 & 0.4418 & 0.3093 & 0.0884 & 0.1104 & 0.1546 \\
0.4418 & 0.4197 & 0.2651 & 0.0441 & 0.1767 & 0.0441 \\
\hline
\end{tabular}

3 do gabarito quando sobrepostos aos dentes do Grupo 1 foram chamados de T1, T2 e T3 (Tofflemire) e U1, U2 e U3 nos dentes do Grupo 2 (Unimatrix). Com esse mesmo software a imagem foi tratada diminuindo-se a opacidade da foto. (Figura 3).

Posteriormente, esse gabarito foi sobreposto em relação às outras fotos, de ambos os grupos, utilizando-se também o software Adobe ${ }^{\circledast}$ Photoshop Acrobat (Figura 4). Servindo-se de um software para medição (Image Pró Plus ${ }^{\odot}$ 4.1.0.0 do fabricante The Proven Solution ${ }^{\circ}$ ) foi aferida a distância entre os pontos determinados na coroa do dente-padrão e dos dentes restaurados. A distância entre os pontos foi aferida em milímetros, fosse essa diferença sobrecontorno ou subcontorno. (Figura 5). Todas as análises ocorreram no Laboratório de Análise Digital em Odontologia da Universidade Ibirapuera (LAIDO).

As diferenças entre os pontos foram anotadas em uma planilha eletrônica Excel $^{\circledR}$ Microsoft $^{\circledR}$ (Tabela 1). As diferenças entre os pontos em cada região da coroa foram analisadas com o teste ANOVA (Tabela 2) e teste complementar de Newman-Keuls (Tabela 3) ao grau de significância de $5 \%$.

\section{RESULTADOS}

A Hipótese de Nulidade a ser testada (Ho) é: não há diferença entre os contornos proximais de restaurações em resina composta comparando-se o sistema de matriz de aço Tofflemire e o de matriz Unimatrix.

A Hipótese Alternativa (H1) é: existe diferença entre as condiçóes avaliadas.

Iniciou-se a análise estatística com uma verificação da homogeneidade e normalidade das amostras, que foi realizada pelo teste de aderência à curva normal e teste de homogeneidade de Cochran, por meio do Software GMC versão 2002. Pelo teste realizado, as amostras foram consideradas com distribuição normal e homo-

Tabela 2 -Resultado da análise estatística ANOVA

\begin{tabular}{|c|c|c|c|}
\hline $\begin{array}{l}\text { FONTES DE } \\
\text { VARIAÇĀO }\end{array}$ & GL & SQ & QM \\
\hline Tratamentos & 5 & 0.969 & 0.194 \\
\hline \multirow[t]{2}{*}{ Erro } & 54 & 0.518 & 0.01 \\
\hline & --- & --- & --- \\
\hline $\mathrm{F}=$ & 20.1987 & --- & --- \\
\hline$(\mathrm{p})=$ & 0 & --- & -- \\
\hline Média $(\mathrm{T} 1)=$ & 0.4484 & -- & --- \\
\hline Média $(\mathrm{T} 2)=$ & 0.4219 & --- & --- \\
\hline Média $(\mathrm{T} 3)=$ & 0.3071 & --- & --- \\
\hline Média $(\mathrm{U} 1)=$ & 0.1259 & -- & --- \\
\hline Média $(\mathrm{U} 2)=$ & 0.1723 & -- & --- \\
\hline \multirow[t]{2}{*}{ Média $(\mathrm{U} 3)=$} & 0.1679 & --- & --- \\
\hline & --- & --- & -- \\
\hline Newman-Keuls: & Q & (p) & --- \\
\hline Médias $(\mathrm{T} 1 \mathrm{a}$ T2) $=$ & 0.8563 & $>0.05$ & --- \\
\hline Médias $(\mathrm{T} 1 \mathrm{a}$ T3 $)=$ & 4.5633 & $<0.01$ & --- \\
\hline Médias $(\mathrm{T} 1$ a U1) $=$ & 10.4096 & $<0.01$ & --- \\
\hline Médias $(\mathrm{T} 1$ a U2) $=$ & 8.9127 & $<0.01$ & --- \\
\hline Médias (T1 a U3) $=$ & 9.0554 & $<0.01$ & --- \\
\hline Médias $(\mathrm{T} 2 \mathrm{a}$ T3) $=$ & 3.7071 & $<0.05$ & --- \\
\hline Médias $(\mathrm{T} 2$ a U1) $=$ & 9.5534 & $<0.01$ & --- \\
\hline Médias (T2 a U2) = & 8.0565 & $<0.01$ & --- \\
\hline Médias (T2 a U3) = & 8.1991 & $<0.01$ & --- \\
\hline Médias $($ T3 a U1) $=$ & 5.8463 & $<0.01$ & --- \\
\hline Médias (T3 a U2) = & 4.3494 & $<0.01$ & --- \\
\hline Médias $($ T3 a U3) $=$ & 4.492 & $<0.05$ & --- \\
\hline Médias (U1 a U2) = & 1.4969 & $>0.05$ & --- \\
\hline Médias $(\mathrm{U} 1$ a U3) $=$ & 1.3543 & $>0.05$ & --- \\
\hline Médias $(\mathrm{U} 2$ a U3) $=$ & 0.1427 & $>0.05$ & --- \\
\hline
\end{tabular}


Costa TA, Raitz R, Belan LC, Matson MR. Análise do contorno da face proximal obtido em restaurações classe ii de resina composta utilizando-se dois tipos diferentes de matrizes metálicas. Revista de Odontologia da Universidade Cidade de São Paulo 2009 jan-abr; 21(1): $31-7$

Tabela 3 -Média dos grupos em milímetros

\begin{tabular}{|c|c|c|c|c|c|c|}
\hline & $\mathrm{T} 1 \quad(0,4484)$ & T2 $(0,4219)$ & T3 $(0,3071)$ & U1 $(0,1259)$ & U2 $(0,1723)$ & U3 $(0,1679)$ \\
\hline U1 $(0,1259)$ & significante & significante & significante & & n.s. & n.s. \\
\hline U2 $(0,1723)$ & significante & significante & significante & n.s. & & n.s. \\
\hline U3 $(0,1679)$ & significante & significante & significante & n.s. & n.s. & \\
\hline T1 $(0,4484)$ & & n.s. & significante & significante & significante & significante \\
\hline T2 $(0,4219)$ & n.s. & & significante & significante & significante & significante \\
\hline T3 $(0,3071)$ & significante & significante & & significante & significante & significante \\
\hline
\end{tabular}

Onde: Significante = diferença estatisticamente significante; n.s. = sem diferença estatisticamente significante

gênea, o que permitiu uma análise de variância de fator único (ANOVA-1 FATOR).

Se a hipótese de nulidade fosse aceita poder-se-ia dizer que as diferenças entre as médias obtidas se devem apenas ao acaso. Caso a hipótese de nulidade fosse rejeitada poder-se-ia dizer que existe diferença estatisticamente significante entre as amostras. Ou seja, as diferenças entre as médias dos grupos avaliados não seriam ocasionais, mas relacionadas aos sistemas de matriz.

O resultado da ANOVA, bem como as interaçóes entre os grupos avaliados, encontram-se na Tabela 2. Nela, pode-se verificar que houve diferença estatisticamente significante, portanto se aceitou a hipótese alternativa (H1).

$\mathrm{Na}$ Tabela 3, observam-se as médias dos grupos em milímetros e interaçóes com o grau de significância. Pode-se verificar que houve diferença estatisticamente significante entre os grupos:T1 x U1;T2 x U2;T3 x U3. Ao se comparar os pontos de interesse dentro do mesmo grupo as diferenças não foram significantes.

\section{DISCUSSÃO}

O contato proximal tem importante papel protegendo o periodonto contra injúrias devidas à impactação alimentar. A morfologia da face oclusal e o contato com os dentes adjacentes têm função de desviar o bolo alimentar para a região bucal e/ou lingual dos dentes durante a mastigação. Dörfer et al. ${ }^{2}$ (2000) afirmam que, se o contato proximal for forte o suficiente, irá resistir a essa separação dos dentes durante a mastigação, e a impactação alimentar não ocorrerá. A perda do contato proximal provoca inclinaçóes dos dentes, distúrbios oclusais relacionados ao dente antagonista e facilidade de retenção de placa e comida (Peumans et al. ${ }^{6}, 2001$ ).

Para auxiliar na confecção do contato proximal e com a intenção de superar ou contornar tais problemas, várias técnicas são sugeridas, como pré encunhamento, utilização de matrizes convencionais ou seccionadas com formato proximal preestabelecido e ultrafina, insertos cerâmicos e pontas plásticas adaptáveis ao fotopolimerizador como o Light-tip e uso de instrumentos desenvolvidos para obtenção de forma e contato proximal como o Contact-Pro (Chain e Baratieri ${ }^{1}$, 1998).

$\mathrm{O}$ uso de matriz de aço com algum tipo de retentor (ex: Tofflemire) fornece uma boa adaptação gengival, mas cria um volume excessivo, deixando deficiente o contorno proximal fisiológico do dente. Usar tais matrizes que deixam excessos ou falta de contorno aumenta o tempo

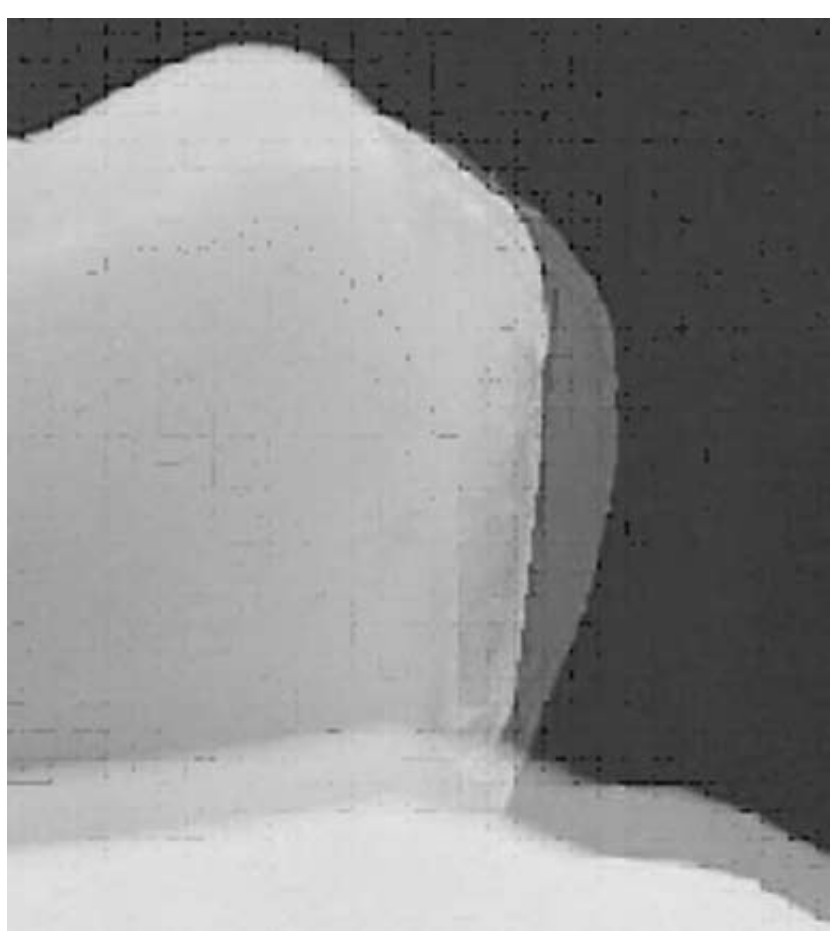

Figura 6: "Aspecto tofflemire" da restauração 
Costa TA, Raitz R, Belan LC, Matson MR. Análise do contorno da face proximal obtido em restaurações classe ii de resina composta utilizando-se dois tipos diferentes de matrizes metálicas. Revista de Odontologia da Universidade Cidade de São Paulo 2009 jan-abr; 21(1): $31-7$

clínico, pois há que se tentar contornar tais problemas. Keogh e Bertolotti ${ }^{3}$ (2001) afirmam que a matriz seccionada minimiza esses defeitos, propiciando um contorno próximo do ideal sem volume excessivo.

Keogh e Bertolotti ${ }^{3}$ (2001) afirmam que historicamente várias técnicas vêm sendo usadas para melhorar o aspecto "Tofflemire" do contato das restauraçóes, cujo contato fica próximo da crista marginal. Esse tipo de contato pode contribuir para o acúmulo de resíduos alimentares pelas faces vestibulares e linguais do dentes restaurados. O contorno proximal em uma restauração direta depende da forma da matriz, especialmente quando essa forma é criada por brunimento quando a matriz já está no lugar. Contudo, esse tipo de brunimento resulta em um contato anatomicamente deficiente.

A avaliação dos resultados desse experimento mostrou que as médias dos valores dos pontos analisados nos dentes do Grupo 1 foram estatisticamente diferentes daquelas analisadas nos dentes do Grupo 2. Isso mostra que as diferenças entre as médias dos grupos não são ocasionais, mas relacionadas aos sistemas de matriz. Quando comparados com o gabarito, os dentes do grupo 1 tiveram no ponto T1 uma média de $0,4484 \mathrm{~mm}$; no ponto T2 média de 0,4219 e no ponto T3 média de 0,3071 . Já os dentes do Grupo 2 tiveram no ponto U1 uma média de $0,1259 \mathrm{~mm}$; no ponto $\mathrm{U} 2$ média de 0,1723 e no ponto U3 média de 0,1679 . Comparando-se os pontos dos dois sistemas de matriz, na mesma regiáo (cervical, terço médio ou oclusal) os dentes do Grupo 1 sempre obtiveram valores maiores do que o Grupo 2, caracterizando um contorno proximal inadequado. Quando as regióes dos pontos foram comparadas dentro de cada grupo, ou seja, T1 com T2 com T3 e U1 com U2 com U3 não foram verificadas diferenças significativas, o que permite dizer que as matrizes metálicas, nos dois sistemas, defletiram proporcionalmente tanto na regiáo cervical como terço médio e oclusal.

Esses resultados corroboram o estudo de Peunans et al. ${ }^{6}$ (2001). Nesse experimento, quatro tipos de resina composta condensáveis foram comparados com um único tipo de resina convencional e três tipos de matrizes: convencional, seccionada e de poliéster. Em relação à firmeza do contato, a matriz seccionada teve resultados significantemente melhores quando comparada aos outros sistemas de matrizes. O tipo de resina, convencio- nal ou condensável, não interferiu na força do contato proximal, especialmente quando a matriz seccionada foi usada.

A maioria dos dentes de ambos os grupos tiveram um subcontorno quando comparados ao gabarito. Contudo, a simulação montada neste estudo foi feita em um manequim sem simulação da membrana periodontal; talvez na boca houvesse uma melhora desse contorno com essa técnica. A diferença entre aos pontos T3 e U3 foi menor do que quando comparados os pontos T1 X U1 e T2 X U2. Isso ocorreu em razão de os dois sistemas de matrizes terem sido usados com cunha interproximal. $\mathrm{O}$ uso da cunha fez com que houvesse uma vedação na região cervical entre a matriz e o dente, causando assim um contorno cervical quando utilizado o sistema de matriz Tofflemire.

O sistema de matriz seccionada proporcionou um contorno adequado, ou seja, por mais que a restauração em resina composta não fique com contato proximal adequado, o contorno proximal fica. Isto se deve ao fato da matriz seccionada ser em forma de "feijão" e com a concavidade na parte interna, anatomicamente melhor do que a superfície plana da matriz convencional. Além disso, o anel retentor da matriz a mantém no lugar, proporcionando também uma separação entre os dentes (Lowe $^{5}$, 2004). O mesmo afirmam Keogh e Bertolotti ${ }^{3}$ (2001). Para eles, a matriz seccionada com o anel retentor é o método mais eficiente para a realização de ótimos contatos e contornos proximais sob várias condiçóes.

Apesar dos dentes que foram restaurados terem ficado sistematicamente sem o ponto de contato com o dente adjacente, os contornos obtidos com o sistema de matriz seccionada foram mais congruentes com o perfil do dente íntegro do que os contornos proximais obtidos com o sistema de matriz Tofflemire.

\section{CONCLUSÃO}

Este estudo "in vitro", de acordo com a metodologia empregada, demonstrou que a qualidade do contorno proximal em restauraçóes Casse II de resina composta é fortemente influenciada pelo tipo de sistema de matriz utilizado.

O sistema de matriz seccionada (Unimatrix) demonstrou um contorno anatômico proximal superior ao formado com o sistema de matriz convencional (Tofflemire). 


\section{REFERÊNCIAS}

1. Chain MC, Baratieri LN. Restaurações estéticas com resina composta em dentes posteriores. 1a. ed. São Paulo: Artes Médicas; 1998.

2. Dörfer CE, von Bethlenfalvy ER, Staehle HJ, Pioch T. Factors influencing proximal dental contact strengths. Eur J Oral Sci 2000 Oct; 108(5): 368-77.

3. Keogh TP, Bertolotti RL. Creating tight anatomically correct interproximal contacts. Dent Clin North Am 2001 Jan; 45(1): 83-102.
4. Klein F, Keller AK, Staehle HJ, Dörfer CE. Proximal contact formation with different restorative materials and techniques. Am J Dent 2002 Aug; 15(4): 232-5.

5. Lowe RA. The use of sectional matrix systems in class II direct composite restorations. Dent Today 2004 Out; 23(10):108, 110-2.

6. Peumans M, van Meerbeek. Asscherickx K, Simon S, Lambrechts P, Vanherle G. Do condensable composites help to achieve better proximal contacts? Dent Mater 2001 Nov; 17(6): 533-41.

Recebido em: 01/07/2008 Aceito em: 16/11/2008 